\title{
Thyroid Hormone Secretion Inhibition
}

National Cancer Institute

\section{Source}

National Cancer Institute. Thyroid Hormone Secretion Inhibition. NCI Thesaurus. Code C41486.

Thyroid Hormone Secretion Inhibition consists of interference with, or restraint of, activities involved in release into the bloodstream by thyroid gland follicular cells of closely related iodine-containing triiodothyronine and thyroxine (thyroid hormones) formed from thyroglobulin that increase the rate of cell metabolism and are essential for central nervous system maturation. Alteration of positive or negative feedback mechanisms can affect hormone release. 